\section{Síndrome de QT largo y taquicardia ventricular en torsade de pointes secundarios a hipopituitarismo: una asociación a tener en cuenta. Caso clínico}

JOSÉ MIGUEL GARCÍA-CASTRO ${ }^{1}$, ANTONIA GARCÍA-MARTÍN² ${ }^{2}$, EMILIO GUIRAO-ARRABAL ${ }^{1}$, PEDRO LUIS CARRILLO-ALASCIO ${ }^{1}$

\section{Long QT syndrome and polymorphic ventricular tachycardia due to hypopituitarism. Report of one case}

Symptoms of hypopituitarism are usually chronic and nonspecific, but rarely the disease can have acute and life threatening manifestations. We report a 53 years old female with a pituitary adenoma that was admitted to our hospital because of syncope. The electrocardiogram showed sinus bradycardia with a prolonged QT interval. Frequent runs of non-sustained polymorphic ventricular tachycardia were noted on telemetry. The patient had a history of severe acute headaches in the previous days and laboratory tests revealed severe secondary hypothyroidism, adrenal insufficiency and a decrease in pituitary hormones. A magnetic resonance imaging of the head showed changes in the size and contrast enhancement of the adenoma. A diagnosis of hypopituitarism secondary to pituitary apoplexy was made and treatment with hydrocortisone and, subsequently, levothyroxine was started. Hormonal disorders such as hypothyroidism, adrenal insufficiency or hypopituitarism should be considered as unusual causes for reversible cardiomyopathy, long QT syndrome and ventricular arrhythmias.

(Rev Med Chile 2017; 145: 941-944)

Key words: Hypopituitarism; Long QT Syndrome; Torsades de Pointes.
'Unidad de Medicina Interna.

Hospital La Inmaculada. Huércal-

Overa, Almería, España.

¿Unidad de Endocrinología y

Nutrición. Complejo Hospitalario

de Granada. Granada, España.

Los autores declaramos no tener conflicto de intereses y no haber recibido ninguna ayuda

financiera.

Recibido el 14 de diciembre de 2016, aceptado el 26 junio de 2017.

Correspondencia a: José Miguel García-Castro. garciacastro82@hotmail.com
E 1 hipopituitarismo consiste en la disminución de la secreción de hormonas hipofisarias, fundamentalmente de las producidas por la adenohipófisis, que puede ser secundaria a enfermedades de la propia hipófisis o a patología del hipotálamo, con disminución de sus hormonas liberadoras. Cuando se afectan todas las hormonas hipofisarias hablamos de panhipopituitarismo. Las manifestaciones clínicas dependerán tanto de la causa del proceso como de la hormona deficiente, el grado de descenso, la velocidad de instauración y la edad del paciente. Los síntomas son generalmente crónicos e inespecíficos como astenia, hiporexia, disfunción sexual o síndrome depresivo de años de evolución, pero en raras ocasiones el déficit hormonal puede instaurarse de forma aguda y suponer una amenaza para la vida ${ }^{1}$.

\section{Caso clínico}

Mujer de 53 años con antecedentes personales de cefalea crónica diaria estudiada, un mes antes del ingreso, mediante resonancia magnética (RMN) cerebral que mostró la presencia de un macroadenoma hipofisario de $18 \mathrm{~mm}$ (Figura 
1a, 1b). Ingresa en el hospital tras presentar un síncope con desconexión súbita del medio, arreactividad a estímulos e hipotonía postural, ocurrido en reposo y autolimitado en pocos minutos. En urgencias la exploración física es normal pero se objetivan hipotensión arterial de $70 / 40 \mathrm{mmHg}$, bradicardia sinusal a $34 \mathrm{lpm}$ y diversas alteraciones electrocardiográficas (Figura 2) como bajos voltajes, extrasístoles ventriculares, aplanamiento difuso de la onda $\mathrm{T}$ e intervalo QT corregido prolongado de $550 \mathrm{mseg}$ (normal hasta $450 \mathrm{mseg}$ ). Durante la monitorización cardiaca la paciente presenta, además, rachas autolimitadas de taquicardia ventricular polimorfa en torsade de pointes que no producen síntomas ni inestabilización hemodinámica, por lo que es tratada con sulfato de magnesio e ingresa en UCI donde se coloca un marcapasos transitorio. En la anamnesis dirigida no refiere síncopes previos ni historia de disnea, dolor torácico o palpitaciones pero destaca un cuadro clínico crónico, de tres meses de evolución, consistente en astenia intensa, ánimo triste, hiporexia, náuseas frecuentes sin vómitos y tendencia a presentar cifras tensionales bajas y diuresis muy abundante, de hasta seis litros diarios. También narra, en los días previos al ingreso, episodio brusco de cefalea intensa frontal acompañada de visión borrosa y vómitos, sin otra clínica neurológica focal, que había sido interpretada como una crisis de migraña. En la analítica inicial destacan hipernatremia ligera con sodio $147 \mathrm{mmol} / \mathrm{L}$ (135-145), hipotiroidismo secundario con FT4 0,07 ng/dL (0,93-1,70), T3 0,44 $\mathrm{pg} / \mathrm{mL}(1,71-3,71)$ y tirotropina o TSH $0,36 \mu \mathrm{UI} /$ $\mathrm{mL}(0,27-1,70)$ e insuficiencia adrenal secundaria con cortisol $1 \mu \mathrm{g} / \mathrm{dL}(6,2-19,4)$ y córticotropina o ACTH 1 pg/mL (0,8-4,2). El estudio hormonal completo muestra descenso de todas las hormonas hipofisarias con hormona luteinizante o $\mathrm{LH}$ $<0,1 \mathrm{mUI} / \mathrm{mL}(7,7-58,5)$, hormona estimulante del folículo o FSH $0,3 \mathrm{mUI} / \mathrm{mL}(25,8-134,8)$, prolactina $1,6 \mathrm{ng} / \mathrm{mL}(4,8-23,3)$, hormona de crecimiento o $\mathrm{GH}<0,2 \mathrm{ng} / \mathrm{mL}(1-16)$ y somatomedina C o IGF - I $32 \mathrm{ng} / \mathrm{mL}$ (87-238). Los niveles de vasopresina o $\mathrm{ADH}$ inferiores a $1 \mathrm{pg} /$ $\mathrm{mL}(1,2-7,6)$, la hipernatremia y la osmolaridad urinaria baja de $198 \mathrm{mmol} / \mathrm{Kg}$ (300-1.300) refuerzan la sospecha de diabetes insípida. Los valores basales obtenidos permiten diagnosticar la existencia de un panhipopituitarismo sin necesidad de pruebas de estímulo. Ante tal hallazgo se realiza una RMN craneal (Figura 1c) donde no se visualizan ni la hipófisis ni el adenoma previo y se aprecia en la silla turca una imagen con señal líquido que parece tener pared, realza con contraste y se acompaña de descenso del quiasma. El caso es valorado por Neurocirugía que descarta indicación de tratamiento quirúrgico. En una nueva anamnesis dirigida la paciente no destaca sintomatología previa de hiperfunción hormonal como crecimiento acral, galactorrea, hirsutismo, hipertensión arterial o alteraciones menstruales o ginecológicas (actualmente con menopausia, nuligesta). Se inicia tratamiento con hidrocortisona intravenosa a dosis de $100 \mathrm{mg}$ cada $8 \mathrm{~h} \mathrm{(al}$ alta $20 \mathrm{mg}$ cada $12 \mathrm{~h}$ vía oral) y levotiroxina oral a dosis $125 \mathrm{mcg}$ diarios, quedando la paciente asintomática con electrocardiograma al alta que muestra un ritmo sinusal con intervalo QT corregido normalizado de $380 \mathrm{mseg}$.

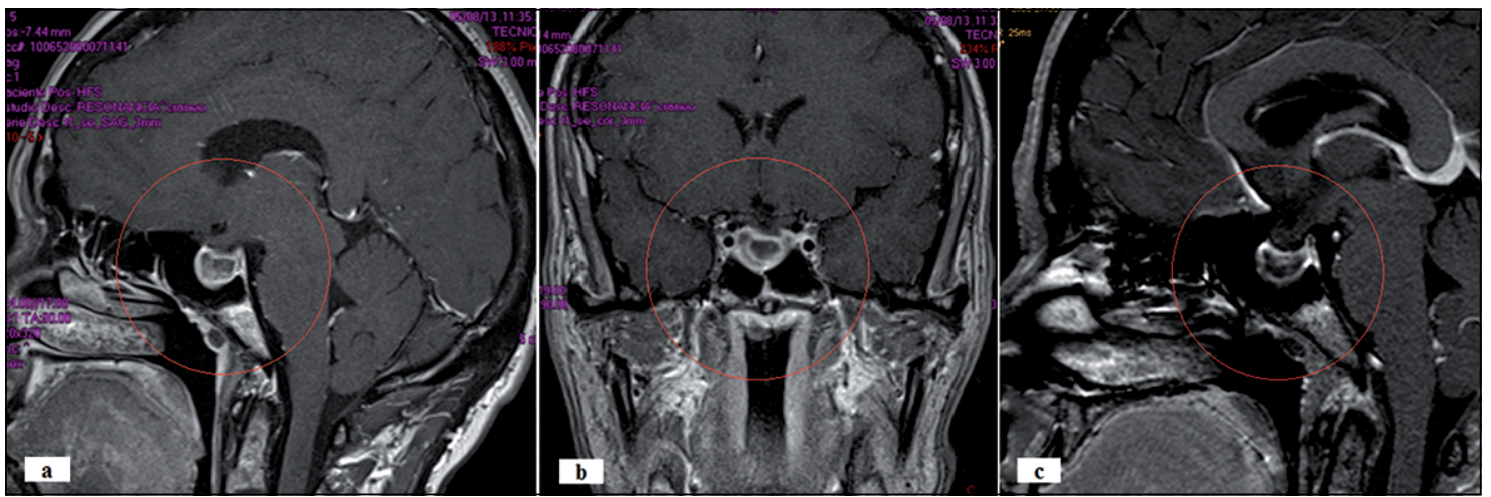

Figura 1. Lesión ocupante del espacio selar en secuencia T1 con captación heterogénea de contraste (a y b). Cambios en el tamaño y en la captación de la lesión tras la apoplejía (c). 


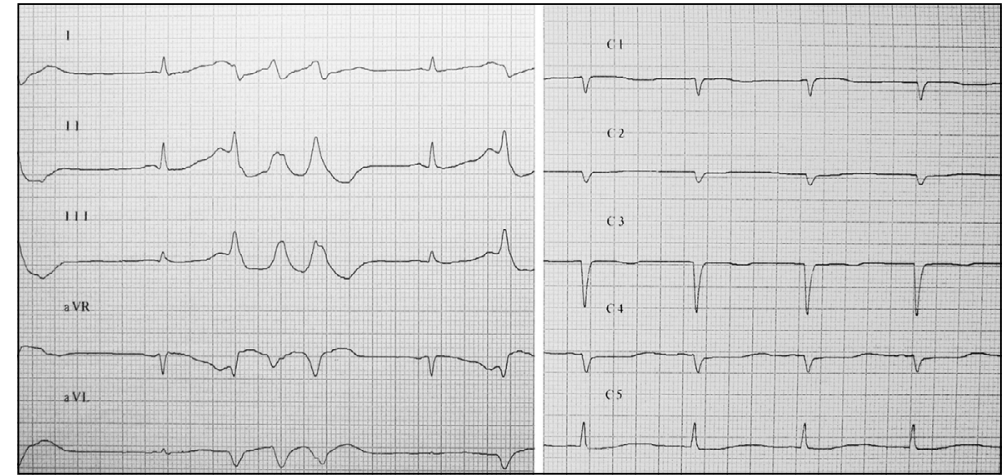

Figura 2. ECG de la paciente. Izquierda: bradicardia con extrasístoles con QRS ancho. Derecha: presencia de bajos voltajes con alteraciones difusas de la repolarización (aplanamiento onda T) y prolongación del QT de hasta 542 mseg.

\section{Discusión}

Nuestra paciente presentó un hipopituitarismo debido a una probable apoplejía hipofisaria sobre un macroadenoma de funcionalidad desconocida, con hipotiroidismo e insuficiencia adrenal secundarios graves que se manifestaron con una bradicardia sinusal, un síndrome de QT largo (SQTL) y una taquicardia ventricular en torsade de pointes.

El hipopituitarismo es una patología de gran interés actual por diversos motivos: el aumento de su incidencia en relación a procesos que suponen un daño cerebral (traumatismos craneales, hemorragia cerebral, ictus, neurocirugía y radioterapia), su asociación con el empeoramiento de la calidad de vida y el aumento de la morbimortalidad cardiovascular de los pacientes y su presentación clínica ocasional en forma de cuadros agudos y graves, potencialmente mortales ${ }^{1}$.

Los tumores hipofisarios (fundamentalmente adenomas) y su tratamiento son la causa más frecuente de hipopituitarismo, provocando más de $60 \%$ de los $\operatorname{casos}^{1}$. La apoplejía hipofisaria implica la destrucción de la glándula como consecuencia de una necrosis isquémica seguida de hemorragia y edema, generalmente sobre un adenoma previo. Cuando es aguda representa una urgencia neuroendocrinológica potencialmente mortal y cursa con cefalea intensa, diplopía, defectos de agudeza y campo visual, vómitos e incluso fiebre o disminución del nivel de consciencia. El desarrollo de hipopituitarismo, transitorio o permanente, es muy frecuente y aunque son más comunes los déficits de GH y gonadotropinas (75-90\% de los pacientes), el hipotiroidismo y la insuficiencia adrenal (40-50\%), son más peligrosos al poder conducir a una situación de shock ${ }^{2}$. Se han des- crito recientemente varios casos en los que el hipopituitarismo se manifiesta por primera vez de forma aguda con una complicación cardiaca grave como un SQTL o una taquicardia ventricular, en ocasiones mortales ${ }^{3-6}$.

Es conocida la asociación del hipopituitarismo con diversas alteraciones electrocardiográficas inespecíficas como bajos voltajes, inversión profunda de la onda $\mathrm{T}$, descenso del segmento ST o prolongación del intervalo QT ${ }^{5,6}$. El SQTL implica una grave alteración de la repolarización ventricular debida a diversas anomalías, congénitas o adquiridas, de los canales iónicos de membrana de los miocitos cardiacos y se asocia a un mayor riesgo de arritmias malignas, como la torsade de pointes, una taquicardia ventricular polimorfa resistente a fármacos antiarrítmicos que, aunque en ocasiones es autolimitada, puede degenerar en fibrilación ventricular y muerte súbita. Entre las causas adquiridas del SQTL destacan fármacos que bloquean el flujo de potasio (principalmente antiarrítmicos de clase IA o III como quinidina, procainamida, sotalol o amiodarona y psicofármacos como haloperidol, fenotiazinas o antidepresivos tricíclicos, también ciertos antihistamínicos, cisaprida, metadona, fluorquinolonas o macrólidos), alteraciones electrolíticas (hipopotasemia, hipomagnesemia e hipocalcemia), bradiarritmias, miocarditis e isquemia cardiaca, lesiones cerebrales (ictus isquémico) y factores dietéticos (desnutrición, dietas hipocalóricas proteicas líquidas), de forma que la susceptibilidad individual podría estar relacionada con polimorfismos genéticos que afectan a la repolarización cardiaca, presentando mayor riesgo el género femenino ${ }^{7}$. También se ha relacionado al SQTL adquirido con diversas alteraciones hormonales, en ausencia de disturbios 
electrolíticos. En este grupo destaca el hipotiroidismo cuyas manifestaciones electrocardiográficas son bien conocidas, incluyendo bradicardia sinusal, ondas P y complejos de QRS de bajo voltaje, retraso en la conducción con bloqueo de rama derecha, aplanamiento o inversión de la onda $\mathrm{T}$ y prolongación del intervalo QT. Así, en el hipotiroidismo, tanto primario como secundario, se ha descrito una incidencia elevada de SQTL (hasta 21-33\%) y una mayor dispersión del intervalo QT, otro factor predictor de arritmias malignas, habiéndose publicado varios casos de torsade de pointes como primera manifestación clínica del hipotiroidismo ${ }^{8}$. Sabemos que la triyodotironina (T3) tiene una influencia fundamental en la contractilidad y frecuencia cardiacas al regular en los miocitos la expresión de genes responsables del transporte de calcio en el retículo sarcoplásmico, del funcionamiento de los canales iónicos o de la bomba sodio-potasio ATPasa o del número y sensibilidad de los receptores de catecolaminas y en el hipotiroidismo se produciría un bloqueo del flujo externo de potasio en los canales de membrana dependientes de voltaje que alteraría de forma grave la repolarización?.

Por otra parte, en el contexto del hipopituitarismo también podrían contribuir al desarrollo de las complicaciones cardiovasculares y del SQTL el resto de déficits hormonales. Se han descrito casos de miocardiopatía dilatada con insuficiencia cardiaca aguda reversible, diversas alteraciones electrocardiográficas y SQTL complicado con taquicardia ventricular, en pacientes con insuficiencia suprarrenal y deficiencia aislada de ACTH. La patogenia podría relacionarse con diversos factores como la hipomagnesemia, la liberación de catecolaminas reactiva a la hipoglucemia o complejas alteraciones en la expresión genética de los canales iónicos de membrana con disturbios electrolíticos a nivel intracelular ${ }^{10,11}$. También se ha observado una mayor prevalencia de SQTL en pacientes varones con hipogonadismo primario o secundario, en los que la alteración electrocardiográfica se corrige durante el tratamiento sustitutivo con testosterona ${ }^{12}$.

En conclusión, es fundamental considerar en el diagnóstico diferencial de pacientes con SQTL, taquicardia ventricular polimorfa o miocardiopatía adquirida con disfunción ventricular izquierda sistólica de etiología incierta la posibilidad de alteraciones hormonales como hipotiroidismo, insuficiencia adrenal o hipopituitarismo ${ }^{5,6,8}$ y realizar de forma precoz en ellos una historia clínica detallada y una evaluación endocrinológica completa. Así, en caso de evidenciarse un déficit hormonal el tratamiento sustitutivo puede ser curativo y evitar estudios y tratamientos invasivos como la realización de un cateterismo cardiaco o la implantación de un desfibrilador ${ }^{3,6,8}$.

\section{Referencias}

1. Schneider HJ, Aimaretti G, Kreitschmann-Andermahr I, Stalla GK, Ghigo E. Hypopituitarism. Lancet 2007; 369 (9571): 1461-70.

2. Català M, Picó A, Tortosa F, Varela C, Gilsanz A, Lucas $\mathrm{T}$, et al. Guía clínica del diagnóstico y tratamiento de la apoplejía hipofisaria. Endocrinol Nutr 2006; 53 (1): 19-24.

3. Lane JD, Keenan NG, Bouloux P, Rogers D. A heart without hormones. Lancet 2012; 379: 1922.

4. Facenda M, Romero-Garrido R, Hernández-Alfonso J, Ramos-López M. Torsade de pointes por QT largo y silla turca vacía. Rev Esp Cardiol 2009; 62 (10): 1193-205.

5. Hajsheikholeslami F, Yazdani S. Sudden cardiac death as a result of neglected hypopituitarism. Int J Endocrinol Metab 2013; 11 (2): 117-9.

6. Kang DG, Kim SE, Park MS, Kim EJ, Lee JH, Park DG, et al. Acquired long QT syndrome with torsades de pointes in a patient with panhypopituitarism due to radiotherapy. Korean Circ J 2013; 43 (5): 340-2.

7. Kramer D, Zimetbaum P. Long-QT syndrome. Cardiol Rev 2011; 19 (5): 217-25.

8. Ellis C, Murray K. When an ICD is not the answer. Hypothyroidism-induced cardiomyopathy and torsades de pointes. J Cardiovasc Electrophysiol 2008; 19 (10): 1105-7.

9. Klein I, Danzi S. Thyroid disease and the heart. Curr Probl Cardiol 2016; 41 (2): 65-92.

10. Eto K, Koga T, Sakamoto A, Kawazoe N, Sadoshima S, Onoyama K. Adult reversible cardiomyopathy with pituitary adrenal insufficiency caused by empty sella. Angiology 2000; 5 1(4): 319-23.

11. Nishizawa S, Nakamura T, Hamaoka T, Matsumuro A, Sawada T, Matsubara H. Lethal arrhythmia and corticosteroid insufficiency. Am J Emerg Med 2009; 27 (9): 1167.

12. Pecori-Giraldi F, Toja M, Filippini B, Michailidis J, Scacchi M, Stramba-Badiale M, et al. Increased prevalence of prolonged QT interval in males with primary or secondary hypogonadism: a pilot study. Int J Androl 2010; 33: 132-8. 